\title{
Thomas Rucker
}

\section{Unterricht, Exemplarität und Subjektivität}

\section{Problemstellung}

Als Theorie des Unterrichts überhaupt ist die Allgemeine Didaktik traditionell insbesondere mit zwei Grundproblemen befasst, nämlich einerseits zu klären, was Unterricht ,ist', und andererseits darüber Auskunft zu geben, wie Unterricht sein soll, d.h. was unter gutem Unterricht verstanden werden kann. Es ist diese zweite Problemstellung, die im Mittelpunkt des vorliegenden Beitrags steht. Diese Ausrichtung ist selbst wiederum im Kontext einer Entwicklung zu sehen, die aktuell sowohl national als auch international beobachtet werden kann, nämlich dass der Unterricht als ein Thema grundlagentheoretischer Überlegungen wiederentdeckt wird. ${ }^{1}$ Einer Allgemeinen Didaktik, die zumindest auch als Reflexionsinstanz begriffen wird, kommt in diesem Zusammenhang u.a. die Aufgabe zu, das Problembewusstsein, das in der Tradition bereits erreicht worden ist, zu erinnern und dieses - auch immer wieder neu - als einen Anspruch zu formulieren, an dem sich aktuelle Beschreibungen von Unterricht zu bewähren haben. ${ }^{2}$

Eine Antwort auf die Frage, was unter gutem Unterricht zu verstehen ist, lautet, diesen als exemplarisches Lehren und Lernen zu begreifen. Was exemplarisches Lehren und Lernen bedeutet, wie dieses gerechtfertigt werden kann, welche theoretischen und praktischen Implikationen eine solche Beschreibung von Unterricht mit sich führt - diese und andere Fragen sind in der Geschichte der Allgemeinen Didaktik insbesondere im Zeitraum zwischen 1950 und 1970 intensiv diskutiert worden.

In dieser Zeit sind auch die zentralen Texte verfasst worden, die das Problemniveau anzeigen, das berücksichtigt werden will, wenn wir uns heute mit dem Thema Exemplarität aus allgemeindidaktischer Perspektive befassen. ${ }^{3}$ Die Bezugnahme auf „das Exemplarische" sollte, wie Josef Derbolav rückblickend schreibt, den "Bildungssinn des Unterrichtswissens" in den Blick rücken und - damit verbunden - das „Anliegen eines ,bildenden Unterrichts' zur Geltung bringen" bzw. erst einmal in Erinnerung rufen. ${ }^{4}$ Exemplarischer Unterricht bedeutet dabei im Kern, am Beispiel und zugleich verallgemeinerbar zu lehren und zu lernen, was u.a. impliziert, die Fülle der Inhalte, die im Unterricht potentiell behandelt werden könnte, auf solche Inhalte zu reduzieren, an denen ein Wissen und Können erarbeitet, angeeignet und erprobt werden kann, das über die jeweiligen Inhalte hinausweist.

Das Interesse an den mit dem Begriff der Exemplarität bezeichneten didaktischen Problemstellungen ist seit den 1970er Jahren merklich zurückgegangen. Damit ist die Gefahr verbunden, hinter ein 
schon einmal erreichtes Problembewusstsein in der Beschreibung von Unterricht zurückzufallen - eines Unterrichts jedenfalls, der darauf gerichtet ist, Schülern Bildung zu ermöglichen. Einer der zentralen Protagonisten der einstigen Debatte - Wolfgang Klafki - hatte deshalb bereits Anfang der 1980er Jahre dafür votiert, die abgebrochene Diskussion wieder aufzugreifen - jedoch ohne sichtbaren Erfolg, wie Klafki rund zwei Jahrzehnte später selbst anmerkt. ${ }^{5}$ Unlängst hat Klaus Zierer erneut einen entsprechenden Versuch unternommen und dabei mögliche Gründe eruiert, warum Exemplarität in der Allgemeinen Didaktik scheinbar in Vergessenheit geraten ist - gerade in neuerer Zeit. Als ein erster Grund wird die heute maßgebliche Outputorientierung im Bildungssystem genannt. Diese habe den Blick von Fragen der Inhaltsauswahl weitestgehend abgelenkt. Die Problemstellungen, die mit dem Begriff der Exemplarität bezeichnet werden, sind aber - primär jedenfalls - solche, die die Auswahl von Unterrichtsinhalten betreffen. Ein weiterer Grund könne, so Zierer, in der Situation der Allgemeinen Didaktik selbst gesehen werden, die in den letzten Jahrzehnten zunehmend in Konkurrenz zu einer empirischen Unterrichtsforschung psychologischer bzw. soziologischer Provenienz einerseits sowie zu florierenden Fachdidaktiken andererseits geraten ist, und dabei einen deutlichen Bedeutungsverlust erfahren hat. ${ }^{6}$ In der Tat spielen im Kontext der empirischen Unterrichtsforschung Fragen der Auswahl von Unterrichtsinhalten eine untergeordnete Rolle, so dass von dieser Seite kaum eine weitere Klärung der mit dem Begriff der Exemplarität bezeichneten Problemstellungen zu erwarten ist. Die Fachdidaktiken hingegen können das Prinzip des Exemplarischen in seiner allgemeinen Bedeutung für schulischen Unterricht nicht klären, sondern bestenfalls unter Voraussetzung dieses Prinzips
Unterricht in einem bestimmten Fach als exemplarisches Lehren und Lernen beschreiben.

Um zu begründen, warum sich die Allgemeine Didaktik auch heute noch dem Thema der Exemplarität widmen sollte, reicht es freilich nicht aus, darauf hinzuweisen, dass eine solche Thematisierung von anderer Seite nur unzureichend geleistet wird bzw. geleistet werden kann. Vielmehr muss deutlich gemacht werden, dass mit dem Begriff des Exemplarischen Problemstellungen bezeichnet werden, die bis heute nichts an Relevanz verloren haben, so dass gute Gründe dafür sprechen, überkommene Beschreibungen exemplarischen Lehrens und Lernens aufzugreifen und weiterzuentwickeln. In diesem Beitrag möchte ich einen entsprechenden Begründungsversuch zur Diskussion stellen.

In einem ersten Schritt beschreibe ich den Kontext, in dem Exemplarität aus allgemeindidaktischer Sicht m.E. diskutiert werden sollte. Hierzu nehme ich insbesondere auf einen neueren Vorschlag Gert Biestas Bezug, den Zweck von Unterricht in dreifacher Hinsicht zu bestimmen, nämlich als Qualifikation, Sozialisation und Subjektwerdung (1.). In einem zweiten Schritt erläutere ich den Grundgedanken exemplarischen Lehrens und Lernen und arbeite ich insbesondere die Funktion heraus, die dem Prinzip des Exemplarischen im Kontext eines Unterrichts mit Bildungsanspruch zukommt. Die These lautet, dass mit dem Begriff des Exemplarität die Form von Inhalten bezeichnet werden kann, die gegeben sein muss, wenn Unterricht dem Anspruch der Bildung Rechnung tragen soll, nämlich Heranwachsende für ein Leben in Selbstbestimmung freizusetzen (2.). In einem dritten Schritt gehe ich schließlich der Frage nach der Kompatibilität zwischen Beschreibungen exemplarischen Lehrens und Lernens sowie dem Anspruch nach, Schüler in ihrer 
Entwicklung als Subjekte zu unterstützen. Dabei verteidige ich zum einen Beschreibungen exemplarischen Lehrens und Lernens gegenüber dem Einwand, dass in einem entsprechenden Unterricht der Anspruch der Subjektwerdung nicht jedenfalls nicht hinreichend - berücksichtigt werde. Zum anderen argumentiere ich dafür, dass überkommene Beschreibungen exemplarischen Lehrens und Lernens hin zu einer Beschreibung eines erziehenden Unterrichts weiterentwickelt werden sollten, in dem Heranwachsende nicht nur zu elementarem Wissen und Können geführt, sondern diese darüber hinaus zu eigenen Positionsbestimmungen veranlasst werden (3.).

\section{Das dreifache Telos guten Unterrichts}

Ist von einem Unterricht mit Bildungsanspruch die Rede, so steht damit nicht - jedenfalls nicht primär - die Frage im Mittelpunkt, was Unterricht ,ist', sondern vielmehr die Frage, wie Unterricht sein soll, damit Bildung möglich wird. Auch ein in diesem Sinne normativer Entwurf kommt freilich nicht ohne einen Begriff von Unterricht aus. Wenn ich im Folgenden von Unterricht spreche, so meine ich damit eine spezifische Grundform pädagogischen Handelns, die stets von Bedeutung ist, wenn Menschen pädagogisch miteinander umgehen. Schulunterricht ist dabei nur eine Erscheinungsform von Unterricht neben anderen. Das Kriterium, anhand dessen Unterricht von alternativen Grundformen pädagogischen Handelns unterschieden werden kann, lautet, dass das Miteinanderumgehen von Erzieher und ZuErziehendem über ein ,Drittes' vermittelt ist, das als Wissen und Können tradiert und angeeignet werden soll. ${ }^{7}$

Was ist unter gutem Unterricht zu verstehen? Hierbei handelt es sich um eine
Frage, die alles andere als leicht zu beantworten ist - dies jedenfalls dann, wenn man nicht der Versuchung erliegt, guten Unterricht vorschnell mit effektivem oder effizientem Unterricht gleichzusetzen. ${ }^{8}$ Die Schwierigkeiten beginnen schon damit, dass unklar ist, ob die Allgemeine Didaktik als Wissenschaft überhaupt dazu in der Lage ist, normative Entwürfe von Unterricht zu entwickeln, ohne dabei ihren Wissenschaftscharakter einzubüßen. Ich kann diese Frage hier nicht ausführlicher diskutieren $^{9}$, gehe aber im Folgenden von der Annahme aus, dass es möglich ist, normative Problemstellungen, die mit Unterricht verbunden sind, mit wissenschaftlichem Anspruch zu bearbeiten, ohne sich dabei auf eine Beobachterposition zurückzuziehen bzw. zurückziehen zu müssen. ${ }^{10}$ Normative Entwürfe in diesem Sinne müssen bestimmten Erwartungen genügen, die es erlauben, sie von Beschreibungen zu unterscheiden, denen zu Recht ein moralisierendes Pathos vorgeworfen wird (Formulierung einer Problemstellung, Offenlegung der in Anspruch genommenen Voraussetzungen, Klärung der verwendeten Begriffe, argumentative Problematisierung von Alternativpositionen etc.).

Gert Biesta hat unlängst einen Vorschlag unterbreitet, was unter gutem Unterricht verstanden werden kann. Biesta votiert dafür, die eingewöhnte Prämisse aufzugeben (zumindest aber vorübergehend einzuklammern), dass Unterricht die Aufgabe besitze, Lernen zu initiieren und zu unterstützen. Der Vorschlag lautet, Unterricht und Lernen gleichsam zu entkoppeln, d.h. die scheinbar omnipräsente Sprache des Lernens (,Lerner', ,Lernbegleiter', ,Ort des Lernens' etc.) zurückzuweisen, und sich stattdessen um alternative Beschreibungen von Unterricht zu bemühen. Dass die Sprache des Lernens in diesem Zusammenhang nur scheinbar eine angemessene Sprache ist, zeigt allein schon 
der Umstand, dass Unterricht es nicht einfach mit der Ermöglichung von Lernen zu tun hat, sondern vielmehr darauf gerichtet ist, dass Heranwachsende bestimmte Inhalte lernen, und zwar zu spezifischen Zwecken sowie unter der Führung durch einen Lehrer. ${ }^{11}$ Eine Theorie des Unterrichts steht deshalb u.a. vor der Herausforderung, das Problem der Aufgabe, des Inhalts und der Lehrer-Schüler-Beziehung zu thematisieren. In diesem Zusammenhang spricht Biesta dem Problem der Aufgabe von Unterricht zu Recht eine herausragende Bedeutung zu. Wie wollte man etwa sinnvoll zu Fragen der Auswahl von Unterrichtsinhalten Stellung nehmen, ohne die Frage geklärt zu haben, wozu Unterricht eigentlich da ist? ${ }^{12}$

Der Vorschlag lautet, das Telos des Unterrichts als plural zu begreifen. Das bedeutet, dem Unterricht kommt nicht nur eine Aufgabe zu, sondern deren drei, die Biesta als Qualifikation (qualification), Sozialisation (socialization) und Subjektwerdung (subjectification) bezeichnet. ${ }^{13}$ Der Begriff der Qualifikation bezeichnet in diesem Zusammenhang die Aufgabe, Heranwachsenden dabei zu helfen, sich das Wissen und Können anzueignen, das Voraussetzung dafür ist, um bestimmte Tätigkeiten in einer Gesellschaft ausüben zu können. Qualifikation in diesem Sinne ist gleichsam das Kerngeschäft von Unterricht. Doch kommt diesem darüber hinaus auch die Aufgabe der Sozialisation zu, worunter Biesta die Einführung der Heranwachsenden in die Werte und Normen versteht, die in einer Gesellschaft gelten. Damit ist freilich nicht gemeint, Sozialisation sei allein die Aufgabe von Unterricht. Die These lautet vielmehr, dass auch dem Unterricht die Aufgabe zukommt, Heranwachsende in eine gegebene gesellschaftliche Ordnung einzuführen. Im deutschen Sprachraum wird diese Aufgabe oftmals unter dem Namen, erziehender Unterricht' thematisiert. Als ,erziehend' gilt dann ein Unterricht, der über die Vermittlung und Aneignung tradierten Wissens und Könnens hinausgeht und dieses mit Werten und Normen in Verbindung bringt, die in einer Gesellschaft als maßgeblich angesehen werden. Dahinter steht die (richtige) Annahme, dass das Wissen und Können, das im Unterricht vermittelt und angeeignet werden soll, nicht notwendigerweise einen Bezug zur Lebensführung der Heranwachsenden aufweist. Allerdings wird in besagten Beschreibungen aus diesem Umstand der (falsche) Schluss gezogen, dass die Vermittlung und Aneignung tradierten Wissens und Könnens allein um eine Vermittlung und Aneignung tradierter Werte und Normen ergänzt werden müsste. Ein solcher Schluss ist deshalb problematisch, weil damit die Gefahr verbunden ist, dass eine dritte Aufgabe von Unterricht aus dem Blick gerät, nämlich dem Einzelnen die Möglichkeit zu eröffnen, das eigene Leben selbstbestimmt zu führen. ${ }^{14}$ Es ist vor allem diese Aufgabe, die in der Tradition der modernen Pädagogik seit jeher besondere Aufmerksamkeit auf sich gezogen hat, und die Biesta als die Aufgabe der Subjektwerdung bezeichnet. In theoretischer Hinsicht stellt sich hier die Frage, wie ein Unterricht beschrieben werden kann, in dem Heranwachsende als Subjekte adressiert werden, die eine eigene und in diesem Sinne durch nichts ersetzbare Perspektive in die Welt hineinbringen. ${ }^{15}$

Biestas Vorschlag, die Aufgabe von Unterricht zu bestimmen, ist freilich nicht neu, wenn wir uns der Problemgeschichte versichern. Was Biesta die Aufgabe der Subjektwerdung nennt, steht in unverkennbarer Nähe zu dem, was in der Allgemeinen Didaktik als der Bildungsanspruch von Unterricht bekannt ist. In diesem Sinne macht z.B. Alfred Schäfer explizit darauf aufmerksam, dass die „Verfügung von Wissen" noch nicht bedeutet, "dass man von Bildung sprechen könnte" 16 Stattdessen sei zu berücksichtigen: „Erst 
wenn das Verhältnis zum Gelernten ins Blickfeld rückt, lassen sich kategorial Übergänge vom Lernen zur Bildung vorstellen"17. Vor diesem Hintergrund mag es durchaus irritieren, dass Biesta dem Bildungsbegriff kritisch gegenübersteht. Dies hat aus meiner Sicht insbesondere damit zu tun, dass Biesta Bildung als Entwicklung von rationaler Autonomie begreift - und damit übersieht, dass die Figur eines Sich-Verhaltens des Einzelnen zum Gelernten für unterschiedliche Ausdeutungen offen ist. Der Bildungsbegriff sei, so Biesta, mit einer spezifischen Auffassung darüber verbunden, was es bedeutet, ,Mensch`zu sein, nämlich eine Person, die dazu bereit und fähig ist, ein Leben zu führen, das „ausschließlich auf rationalen Überzeugungen beruht". Aufgrund dieses anthropologischen ,Haltepunktes' werde die Offenheit, die für einen Unterricht mit Bildungsanspruch traditionell als charakteristisch angesehen wird, unmittelbar limitiert, indem „Bildung als ein Übergang - manche würden es auch eine Befreiung nennen - von einer an Tradition orientierten hin zu einer rationalen Lebensführung" beschrieben wird. Indem hier eine spezifische Auffassung von einem gelingenden Leben als die allein ,richtige" Auffassung vorausgesetzt werde, wird alternativen Entwürfen (z.B. eines an Tradition orientierten Lebens) "Ungerechtigkeit" zuteil. ${ }^{18}$

Sollte diese Beschreibung zutreffen, so hätte dies weitreichende Konsequenzen etwa was die Differenz von Bildung und Sozialisation bzw. die Differenz zwischen einem Unterricht als Bildungs- und einem Unterricht als Sozialisationshilfe betrifft. Hat man nämlich erst einmal erkannt, dass es selbst eine Tradition ist, sich Traditionen gegenüber kritisch zu verhalten, so müsste es konsequenterweise als Sozialisation in eine bestimmte Form der Lebensführung begriffen werden, wenn Heranwachsende lernen, Traditionen auf den Prüfstand zu stellen. Pointiert formuliert: Ein an der
Ermöglichung von Bildung orientierter Unterricht ist "nur eine weitere Form von (moderner, westlicher) Sozialisation"19.

Ein Unterricht, der mit dem Anspruch der Subjektwerdung Ernst macht, d.h. den Einzelnen als Subjekt adressiert, verlangt danach, jegliche Bezugnahme auf eine spezifische Auffassung von einem gelingenden Leben zunächst einmal zu suspendieren. „Wir sollten nicht versuchen, vor dem Ereignis zu urteilen - wir sollten nicht versuchen, zu bestimmen, was Schüler und Kinder und Neuankömmlinge sein sollen, bevor sie ankommen. Wir sollten sie erst ankommen lassen und erst dann ein Urteil fällen" ${ }^{20}$. Wie bereits angedeutet, halte ich es jedoch für zu kurz gegriffen, Beschreibungen von Unterricht, die bildungstheoretisch ausgerichtet sind, in toto zu unterstellen, der Einzelne würde darin auf eine rationale Lebensführung verpflichtet werden. Einen Unterricht mit Bildungsanspruch etwa als Aufforderung zur Prüfung von Geltungsansprüchen zu beschreiben ${ }^{21}$, ist nicht gleichbedeutend damit, Heranwachsende auf diese Form der Lebensführung hin festzulegen. Gemeint ist damit zunächst einmal nur, dass Heranwachsende dazu aufgefordert werden, Geltungsansprüche nicht unbefragt zu akzeptieren, sondern sich hierzu in ein Verhältnis zu setzen (wozu dann konsequenterweise auch der Anspruch zu zählen wäre, eine rationale Lebensführung sei die allein ,richtige' Form der Lebensführung). Der Ausgang dieses Sich-Verhaltens ist offen, und muss offen bleiben, soll der Einzelne als Subjekt adressiert werden. Biesta greift in diesem Zusammenhang zustimmend Benners Formulierung von der ,Aufforderung zur Selbsttätigkeit' auf, um deutlich zu machen, was unter einem Unterricht zu verstehen ist, in dem "Individuen dazu veranlasst werden, als Subjekte zu ,erscheinen“"22. Doch ist damit kein grundlegender Unterschied zu dem benannt, was ich als Aufforderung zur 
Prüfung von Geltungsansprüchen bezeichne. Beide Formulierungen rücken lediglich unterschiedliche Facetten dessen in den Blick, was unter einem Unterricht mit Bildungsanspruch $\mathrm{zu}$ verstehen ist. Im ersten Fall (,Aufforderung zur Selbsttätigkeit') wird betont, dass der Einzelne dazu veranlasst werden soll, eine eigene Position unter Berücksichtigung alternativer Positionen zu entwickeln. Im zweiten Fall (,Aufforderung zur Prüfung von Geltungsansprüchen') wird hervorgehoben, dass der Einzelne daraufhin angesprochen werden soll, sich unter Berücksichtigung alternativer Positionen selbst zu bestimmen.

Aus der Perspektive einer bildungstheoretisch orientierten Allgemeinen Didaktik kann deshalb festgehalten werden: Guter Unterricht bedeutet nicht einfach, Heranwachsende zu qualifizieren und diese in tradierte Wert- und Normsysteme einzuführen, sondern ist darüber hinaus auch und vor allem darauf gerichtet, Schülern die Möglichkeit zu eröffnen, ihr Leben selbstbestimmt führen zu lernen. Ein Unterricht mit Bildungsanspruch geht in diesem Sinne nicht darin auf, dass $\mathrm{He}$ ranwachsende etwas lernen; sie sollen darüber hinaus auch die Möglichkeit erhalten, sich zu Gelerntem zu verhalten, und zwar ohne dass dieses Sich-Verhalten bereits vorab auf einen bestimmten Umgang mit dem Gelernten hin finalisiert ist. Damit ist wiederum nicht gemeint, dass Heranwachsende nicht in überkommene Werte und Normen eingeführt werden sollten. Im Gegenteil: Lehrer präsentieren und repräsentieren diese Werte und Normen, sie sollen dies aber nicht so tun, dass die damit verbundenen Geltungsansprüche durchgesetzt werden. Die Heranwachsenden sollen vielmehr zur Prüfung von Geltungsansprüchen aufgefordert werden, damit diese die Möglichkeit erhalten, ihre Perspektive ins Spiel zu bringen, um in der Auseinandersetzung mit tradierten Werten und Normen eine eigene Werteorientierung entwickeln und diese schließlich auch intergenerationell beraten zu können.

\section{Ort und Funktion des Exemplarischen}

Die vorangegangenen Überlegungen könnten der Eindruck erwecken, dass es letztlich gleichgültig sei, welche Unterrichtsinhalte es sind, die vermittelt und angeeignet werden, solange nur sichergestellt ist, dass die Heranwachsenden die Möglichkeit haben, sich zu Gelerntem zu verhalten. Eine solche Schlussfolgerung konfligiert mit einer These, die für Beschreibungen exemplarischen Lehrens und Lernens zentral ist, nämlich dass gerade nicht jeder Unterrichtsinhalt auch schon als Bildungsinhalt begriffen werden könne. Als Bildungsinhalte seien vielmehr nur solche Inhalte zu bezeichnen, die den Kriterien der Gegenwarts- und Zukunftsbedeutung entsprechen und die darüber hinaus - und das ist hier entscheidend dem Kriterium der Exemplarität genügen. ${ }^{23}$

Die These, die ich im Folgenden erläutern und begründen möchte, lautet nicht nur, dass Exemplarität als ein Auswahlkriterium von Inhalten in die bislang entwickelte Beschreibung eines Unterrichts mit Bildungsanspruch integriert werden kann. Ich möchte vielmehr für die stärkere These argumentieren, dass eine solche Integration vorgenommen werden muss, und zwar deshalb, weil eine Freisetzung der Schüler für ein Leben in Selbstbestimmung nur unter der Voraussetzung sinnvoll beschrieben werden kann, dass die Inhalte, die im Unterricht vermittelt und angeeignet werden, dem Kriterium der Exemplarität entsprechen. Zugespitzt formuliert: Unterrichtsinhalte müssen dem Anspruch der Exemplarität genügen, da ansonsten die Freisetzung der Heranwachsenden für ein Leben in 
Selbstbestimmung nicht plausibel gemacht werden kann. ${ }^{24}$

Um diese These zu erläutern, ist ein Rückgriff auf die Theorie der kategorialen Bildung hilfreich, d.h. auf jene Bildungstheorie, die Wolfgang Klafki in den 1960er Jahren dazu veranlasst hat, potentiell bildenden Unterricht als exemplarisches Lehren und Lernen zu beschreiben. Hierbei wird man zunächst festhalten müssen, dass der Theorie der kategorialen Bildung im Fach aktuell keine "diskursstiftende Rolle $^{\text {"25 }}$ zukommt. Das bedeutet jedoch nicht, dass die Probleme, die in der Theorie der kategorialen Bildung behandelt werden, in bildungstheoretisch-didaktischer Hinsicht an Bedeutung verloren hätten. Vielmehr bin ich mit Dietrich Benner der Auffassung, dass Klafkis „didaktisch orientiertes Verständnis einer doppelten Erschließung" von Mensch und Welt für die „Weiterentwicklung der allgemeinen Didaktik und der Fachdidaktik" sowie für die „Weiterentwicklung der empirischen Bildungsforschung zu einer zumindest auch pädagogisch und erziehungswissenschaftlich argumentierenden Disziplin" von „bleibender Bedeutung" ist. ${ }^{26}$

Ich werde an dieser Stelle keine ausführliche Rekonstruktion der Theorie der kategorialen Bildung vornehmen. ${ }^{27}$ Stattdessen werde ich mich darauf beschränken, die Theorie der kategorialen Bildung mit der Theorie der funktionalen Bildung zu kontrastieren. Dies ist ausreichend, um die These zu verdeutlichen, dass mit dem Begriff des Exemplarität diejenige Form von Inhalten bezeichnet werden kann, die gegeben sein muss, wenn Unterricht dem Anspruch Rechnung tragen soll, Heranwachsende für ein Leben in Selbstbestimmung freizusetzen.

Funktionale Bildungstheorie ist nach Klafki daran erkennbar, dass Bildung als ein Prozess der Entwicklung von Kräften beschrieben wird. Aufgabe von Unterricht ist es, diesen Prozess der
Kräfteentwicklung zu initiieren und zu unterstützen. Die Voraussetzung, auf der eine solche Beschreibung eines potentiell bildungsstiftenden Unterrichts beruht, lautet, dass es menschliche Kräfte gibt, die sich durch Übung an geeigneten Inhalten entwickeln und die anschließend an beliebigen Inhalten in Funktion treten können. Hat eine Person z.B. die Kraft der Phantasie erst einmal entwickelt, so die Annahme, dann ist diese Kraft nicht länger an bestimmte Inhalte gebunden, sondern kann in Bezug auf verschiedene Inhalte zum Einsatz gebracht werden. Unterricht wäre vor diesem Hintergrund in dem Sinne als emanzipativ zu begreifen, als die fortschreitende Entwicklung von Kräften Schüler sukzessive von unterrichtlicher Sorge freisetzt.

Folgt man Klafki, so ist die Theorie der funktionalen Bildung mit dem Problem konfrontiert, das Phänomen nicht erklären zu können, wie jemand, der in einem bestimmten Bereich z.B. eine ausgeprägte Urteilskraft erkennen lässt, in anderen Bereichen kaum zu angemessenen Urteilen über Sachverhalte in der Lage ist. Klafki interpretiert dies als einen Hinweis darauf, dass die unterstellten Kräfte auf der Seite des Heranwachsenden offenbar doch nicht in dem Maße von den Inhalten losgelöst werden können, wie dies im Rahmen funktionaler Bildungstheorie angenommen wird. ${ }^{28}$

Klafkis Theorie der kategorialen Bildung offeriert eine alternative Beschreibung von Bildung und - damit verbunden - eines potentiell bildungsstiftenden Unterrichts. Die These lautet, dass es nicht die Entwicklung von Kräften ist, die eine Freisetzung der Schüler von unterrichtlicher Sorge ermöglicht, sondern ein in der Auseinandersetzung mit bestimmten Inhalten entwickeltes allgemeines Wissen und Können. Es sind diese Kategorien, die - in der Auseinandersetzung mit strukturanalogen Inhalten - den Eindruck erwecken, 
es hätte sich eine Kraft auf der Seite der Heranwachsenden entwickelt. Tatsächlich aber handelt es sich um ein Wissen und Können, das aufgrund seines allgemeinen Charakters nicht auf den Inhalt beschränkt ist, der im Unterricht thematisiert wurde, und das gerade deshalb ein emanzipatorisches Potential besitzt: „Jeder erkannte oder erlebte Sachverhalt auf der objektiven Seite löst im Zögling nicht eine subjektive, ,formale' Kraft aus oder ist Übungsmaterial solcher subjektiven Kräfte [...], sondern er ist - in einem übertragenen Sinne - selbst Kraft, insofern - und nur insofern - er ein Stück Wirklichkeit erschließt und zugänglich macht"29.

Klafki bezeichnet das am Besonderen gewonnene Allgemeine als das Elementare, und das Besondere, an dem Allgemeines gewonnen wird, als das Exemplarische. Beide Begriffe stehen damit in einem irreduziblen Zusammenhang: „Das Pädagogisch-Elementare ist seinem Wesen nach immer ein Allgemeines. Aber nun muß doch sogleich die andere Seite der Sache in den Blick genommen werden: Das Allgemeine wird nur dort wirklich erfaßt, nur dort bildend wirksam, wo es dem Menschen, dem Kinde nicht als solches, nicht abstrakt dargeboten wird, sondern wo es sich als das Prinzip eines Besonderen erweist, wo es im eigenen geistigen Ringen um ein interessierendes Besonderes erfahren und erfaßt wird“30. Die These lautet also: Das Allgemeine, sofern ihm eine selbst- und welterschließende Bedeutung zukommen soll, muss am Besonderen angeeignet, und die Relation von Besonderem und Allgemeinem muss in der Auseinandersetzung mit weiteren besonderen Inhalten in ihrer selbst- und welterschließenden Funktion einsichtig werden. Auf diese Weise können Schüler erkennen, dass z.B. eine am Besonderen gewonnene Einsicht nicht nur das Verstehen des jeweiligen besonderen Inhalts ermöglicht, sondern über diesen hinausweist und einen mehr oder minder weitreichenden Ausschnitt von Welt zugänglich macht. Umgekehrt ist der besondere Unterrichtsinhalt gerade deshalb kein Selbstzweck - mag der jeweilige Inhalt auch Gegenwarts- und Zukunftsbedeutung für Schüler besitzen. Ein Inhalt muss dazu geeignet sein, dass Heranwachsende exemplarisch Allgemeines erschließen können, um als Bildungsinhalt zu gelten, da Schüler nur so lernen können, mit der Relation von Besonderem und Allgemeinem zu operieren.

Vor diesem Hintergrund votiert Klafki für eine unterrichtliche "Konzentration auf das exemplarisch zur Darstellung gelangende Elementare" ${ }^{\text {"31 }}$, ohne dabei jedoch der Frage hinreichend Aufmerksamkeit zu schenken, inwiefern eine solche Konzentration für einen Unterricht, der auf die Freisetzung der Schüler für ein Leben in Selbstbestimmung gerichtet ist, konstitutiv sein soll. Auch die Klafki-Forschung hat bislang kaum etwas dafür getan, die Ausrichtung des Unterrichts auf das ,exemplarisch zur Darstellung gelangende Elementare' in ihrer Bedeutung für eine entsprechende Freisetzung der Heranwachsenden zu klären.

Es findet sich in den Arbeiten Klafkis eine bislang nur wenig besprochene Passage, die m.E. den Weg in die Richtung weist, in der eine entsprechende Verhältnisbestimmung vorgenommen werden kann: „Bildendes Lernen, das die Selbständigkeit des Lernenden fördert, also zu weiterwirkenden Erkenntnissen, Fähigkeiten, Einstellungen führt [...] wird nicht durch reproduktive Übernahme möglichst vieler Einzelkenntnisse-, -fähigkeiten und -fertigkeiten gewonnen, sondern dadurch, daß sich der Lernende an einer begrenzten Zahl von ausgewählten Beispielen [Exempeln] aktiv allgemeine, genauer: mehr oder minder weitreichend verallgemeinerbare Kenntnisse, Fähigkeiten, Einstellungen erarbeitet [...]. Mit Hilfe solcher allgemeinen 
Einsichten, Fähigkeiten, Einstellungen können jeweils mehr oder minder große Gruppen strukturgleicher oder ähnlich strukturierter Einzelphänomene und -probleme zugänglich bzw. lösbar werden"32. Wie ist das zu verstehen? In welcher Beziehung steht exemplarisches Lehren und Lernen zu dem Bildungsanspruch von Unterricht, Schüler für ein Leben in Selbstbestimmung freizusetzen? Das Argument für eine entsprechende Rechtfertigung exemplarischen Lehrens und Lernens kann aus meiner Sicht wie folgt rekonstruiert werden:

1. Guter Unterricht verfolgt nicht allein die Aufgaben der Qualifikation und der Sozialisation, sondern ist darüber hinaus darauf bezogen, Schülern Bildung zu ermöglichen.

2. Ein Unterricht mit Bildungsanspruch ist auf die Freisetzung der Schüler für ein Leben in Selbstbestimmung gerichtet.

3. Eine selbstbestimmte Lebensführung schließt die Emanzipation der Schüler von der unterrichtlichen Sorge durch einen Lehrer mit ein.

4. Emanzipativ ist ein Lernen, in dem Schüler sich ,weiterwirkendes' Wissen und Können aneignen, das es diesen ermöglicht, strukturähnliche Sachverhalte zu verstehen, ohne dabei auf die Hilfe durch einen Lehrer angewiesen zu sein.

5. Die Entwicklung solchen Wissens und Könnens setzt einen Unterricht voraus, in dem Inhalte thematisiert werden, anhand derer exemplarisch Allgemeines erarbeitet, angeeignet und erprobt werden kann.

Hieraus folgt: Unterricht mit Bildungsanspruch ist in inhaltlicher Hinsicht als exemplarisches Lehren und Lernen zu bestimmen.

Der entscheidende Gedanke lautet an dieser Stelle, dass von Selbstbestimmung nur dann sinnvoll gesprochen werden kann, wenn Schüler - zumindest in einem bestimmten Bereich - auf Lehrer nicht länger angewiesen sind, und damit strenggenommen - jedenfalls in dem besagten Bereich - keine Schüler mehr sind. Inhalte, an denen allgemeines Wissen und Können vermittelt und angeeignet werden kann, haben ein emanzipatorisches Potential, insofern Schüler - im Falle einer erfolgreichen Aneignung - von unterrichtlicher Sorge freigesetzt werden. Deshalb ist ein Unterricht mit Bildungsanspruch in inhaltlicher Hinsicht als exemplarisches Lehren und Lernen zu bestimmen.

Die bisherigen Überlegungen dürfen nicht dahingehend missverstanden werden, als sei das Kriterium der Exemplarität den beiden Kriterien der Gegenwarts- und Zukunftsbedeutung übergeordnet. Die drei Kriterien stehen vielmehr in einer nicht-hierarchischen Beziehung zueinander. Was ein Bildungsinhalt ist, kann niemals ,für sich' bestimmt werden, sondern immer nur relational zu bestimmten Schülern sowie den gesellschaftlichen Bedingungen, unter denen diese gegenwärtig aufwachsen und voraussichtlich zukünftig ihr Leben führen werden. Zugespitzt formuliert: „Wer das Pädagogisch-Elementare finden will, muß den suchenden Blick zugleich auf die Wirklichkeit, auf die Inhalte, und auf das Kind richten" ${ }^{\text {"3. }}$. Mehr noch: Nicht weil ein Lehrer in Bezug auf einen Schüler einen Bildungsgehalt ermessen hat, kommt einem Inhalt der Status eines Bildungsinhalts zu. Letztlich entscheidet erst das Werturteil des Schülers darüber, ob es sich bei einem potentiellen auch um einen tatsächlichen Bildungsinhalt handelt, nämlich dann, wenn dieser dem jeweiligen Inhalt Lebensbedeutung zubzw. abspricht. ${ }^{34}$ Diese Einsicht findet sich in Klafkis Arbeiten nicht hinreichend expliziert, worauf Aloysius Regenbrecht bereits frühzeitig aufmerksam gemacht hat: "Solange Bildungsgehalte in den 
Gegenständen aufgesucht und aufgefunden werden - wenn auch als Bedeutungsgehalt für die konkrete Individualität -, ist das Recht und die Notwendigkeit zu eigener Entscheidung aus der Ganzheit personaler Existenz nicht gesehen"35. Ich möchte im Folgenden näher auf diesen Einwand Regenbrechts eingehen, der sich auch als ein Votum dafür interpretieren lässt, die Beschreibung eines Unterrichts mit Bildungsanspruch als exemplarischem Lehren und Lernen hin zu einer Beschreibung eines erziehenden Unterrichts mit Bildungsanspruch weiterzuentwickeln.

\section{Exemplarität, Subjektivität und Moralität}

Traditionelle Versuche, Unterricht als exemplarisches Lehren und Lernen zu bestimmen, sehen sich seit jeher einer Reihe von Einwänden ausgesetzt. Im Folgenden möchte ich auf einen Einwand eingehen, der noch einmal an den Anfang der Überlegungen zurückführt. Es stellt sich nämlich die Frage, ob und, falls ja, inwiefern eine Beschreibung von Unterricht als exemplarischem Lehren und Lernen tatsächlich kompatibel ist mit dem Anspruch der Subjektwerdung. Der Verdacht einer möglichen Inkompatibilität liegt darin begründet, dass im Falle exemplarischen Lehrens und Lernens - salopp formuliert - bereits feststeht, was am Ende des Unterrichts als Ergebnis herauskommen soll, nämlich ein bestimmtes elementares Wissen und Können. An dieser Stelle könnte man mit Biesta einwenden, „dass ein Unterricht, der auf die Subjektivität des Schülers abzielt, zuallererst riskant ist, denn das Ergebnis des Unterrichts ist unvorhersehbar" ${ }^{\text {"36 }}$, und zwar gerade deshalb, weil dem Einzelnen die Möglichkeit eingeräumt wird, eine Sache anders in den Blick zu nehmen, als diese bislang beschrieben worden ist. ${ }^{37}$
Dieser Einwand findet sich, wie bereits angedeutet, in ähnlicher Form schon in den 1960er Jahren von Regenbrecht formuliert. Dieser erkennt zwar an, dass exemplarischer Unterricht in methodischer Hinsicht als Aufforderung zur Selbsttätigkeit konzipiert wird. Zugleich kritisiert Regenbrecht, dass Aufforderung zur Selbsttätigkeit hier lediglich in einem basalen Sinne verstanden werde, womit dem Anspruch, den Einzelnen als Subjekt zu adressieren, nicht - zumindest aber nicht hinreichend - Rechnung getragen werde: "Gelassen wird ihm die Selbsttätigkeit des Erwerbs - das heißt dann Aktivität im Bildungsprozeß -, genommen ist ihm die Selbsttätigkeit der Entscheidung in der allererst seine Personalität sich zugleich bewährt und gestaltet" ${ }^{\text {38 }}$.

Was lässt sich einem solchen Einwand entgegnen? Aus meiner Sicht kann in mindestens zweifacher Hinsicht hierauf reagiert werden.

Erstens: Exemplarischer Unterricht wird traditionell als genetisch-sokratischer Unterricht konzipiert. ${ }^{39}$ Dieser Umstand findet in Regenbrechts Einwand keine Berücksichtigung. Genetisch heißt ein Unterricht, in dem Schüler dazu veranlasst werden, elementares Wissen und Können in ihrem sachlogischen Aufbau nachzuvollziehen bzw. nachentdecken. Dies schließt insbesondere mit ein, die Heranwachsenden in die Fragen einzuführen, auf die ein bestimmtes Wissen und Können Antworten bietet. ${ }^{40}$ Diese Antworten wiederum sind entweder selbst Methoden, die Menschen entwickelt haben, um bestimmte Fragen zu beantworten, oder Ergebnisse des Einsatzes von Methoden. Hieraus folgt, dass es erforderlich ist, dem "immanent-methodischen Charakter" ${ }^{41}$ tradierten Wissens und Könnens Rechnung zu tragen, indem Schüler die Möglichkeit erhalten, den Weg, der zu einem bestimmten Wissen und Können führt, in vereinfachter Form selbst zu gehen. Ein Inhalt 
wird nicht in fertiger Gestalt präsentiert, sondern die Heranwachsenden werden dazu aufgefordert, die Struktur des jeweiligen Sachverhalts entweder Schritt für Schritt nachzuvollziehen bzw. nachzuentdecken oder diese vom Ergebnis her rückschreitend zu erschließen. ${ }^{4}$

Sokratischer Unterricht bedeutet in diesem Zusammenhang, dass Unterricht als Aufforderung zur Prüfung sachlicher Geltungsansprüche begriffen wird. Eine solche Aufforderung ist für einen genetischen Unterricht nicht notwendigerweise charakteristisch. Dieser kann prinzipiell auch die Form der Unterweisung annehmen: Der sachlogische Aufbau einer Sache wird zwar schrittweise erschlossen, doch steht die Geltung der Sache nicht zur Debatte. Genetisch-sokratisches Lehren ist demgegenüber nicht nur darauf gerichtet, dass Schüler Schritt für Schritt den sachlogischen Aufbau eines Inhalts nachvollziehen bzw. nachentdecken, sondern bedeutet darüber hinaus auch, dass die Heranwachsenden - unter Berücksichtigung ihrer Individuallage - zur Prüfung der Geltungsansprüche aufgefordert werden, die in diesem Zusammenhang eine Rolle spielen. Heranwachsende in diesem Sinne zum "Selberdenken" aufzufordern und - damit verbunden - „zum selbständigen Finden von Erkenntnissen zu verhelfen"43, bedeutet freilich etwas anderes als das, was der von Platon dargestellte Sokrates selbst tut, nämlich Fragen zu stellen, die fast immer schon die Antworten enthalten. Es ist deshalb nicht ohne Grund der Vorschlag gemacht worden, in Beschreibungen eines Unterrichts mit Bildungsanspruch von neosokratischem Unterricht zu sprechen, um deutlicher herauszustellen, dass es in diesem Zusammenhang um die Initiierung und Unterstützung einer Suchbewegung geht, deren Ergebnis in einem bestimmten Sinne offen ist. ${ }^{44}$

Halten wir also fest: Exemplarischer Unterricht ist nicht auf die Durchsetzung von Geltungsansprüchen gerichtet. Als genetisch-sokratischer Unterricht ist dieser vielmehr als eine Einladung an die Schüler zu verstehen, tradiertes Wissen und Können nur dann zu akzeptieren, wenn dieses sich in einer Prüfung tatsächlich als überzeugend erweist. Freilich: Wie die Formulierung vom ,selbständigen Finden von Erkenntnissen' zeigt, wird in Beschreibungen exemplarischen Lehrens und Lernens von der Annahme ausgegangen, dass das jeweilige elementare Wissen und Können von den Schülern als gültig eingesehen werden kann. Das bedeutet aus meiner Sicht aber keineswegs, dass damit der Anspruch der Subjektwerdung unterlaufen werden würde. Im Gegenteil: Indem die Heranwachsenden zur Prüfung von Geltungsansprüchen veranlasst werden, werden sie als Subjekte angesprochen. Unterricht in diesem Sinne zielt nicht - wie der Einwand Regenbrechts nahelegt - auf eine nicht näher spezifizierte Aktivität, sondern auf eigene Urteilsbildung und damit eben nicht auf die unbefragte Aneignung vorgegebener Urteile. Insofern hält exemplarisches Lehren und Lernen prinzipiell die "Möglichkeit zum Widerspruch" offen - dies freilich nicht „im Sinne beliebiger Subjektivismen", sondern unter Berücksichtigung des "Geltungsanspruchs von Wahrheit und Wert"45.

Zweitens: Der Einwand Regenbrechts lässt sich als ein Argument dafür interpretieren, dass Unterricht nur dann sinnvoll als ein Unterricht mit Bildungsanspruch beschrieben werden kann, wenn er zugleich als erziehender Unterricht entworfen wird. Hierunter verstehe ich einen Unterricht, in dem Schüler nicht nur zu elementarem Wissen und Können geführt, sondern diese darüber hinaus auch zu eigenen Werturteilen veranlasst werden, in denen angeeignetes Wissen und Können in seiner Bedeutung für das Leben und Zusammenleben eingeschätzt wird. Ein solcher Begriff von erziehendem Unterricht steht 
in einer Tradition, die auf Johann Friedrich Herbart zurückgeht. ${ }^{46}$ Zentral für diese Tradition ist die Einsicht, dass das Wissen und Können, das im Unterricht vermittelt und angeeignet werden soll, zunächst einmal indifferent ist hinsichtlich der Frage, welche Bedeutung diesem Wissen und Können für ein gelingendes Leben und Zusammenleben zukommt. Erziehender Unterricht wird vor diesem Hintergrund nicht nur als eine Hinführung zu tradiertem Wissen und Können konzipiert, sondern darüber hinaus auch ein ,Hineinziehen" der Heranwachsenden in Situationen, in denen sich diese vor die Aufgabe gestellt sehen, die Bedeutung des Gelernten für das Leben und Zusammenleben zu ermessen. In diesem Sinne wird es in einem erziehenden Unterricht nicht dem Zufall überlassen, ob Schüler die Bedeutung sachlicher Einsichten für die eigene Lebensführung bedenken. Umgekehrt sind die Werturteile, deren Entwicklung initiiert und unterstützt werden soll, über eine Aneignung von Wissen und Können vermittelt. Die Heranwachsenden sollen dazu befähigt werden, im Lichte sachlicher Einsichten Stellung zu nehmen.

Entscheidend ist an dieser Stelle die folgende Überlegung: Fragen des gelingenden Lebens und Zusammenleben sind in modernen Gesellschaften notorisch umstritten, so dass Heranwachsende hier keinen festen ,Halt' an allgemein geteilten Überzeugungen finden können und damit gleichsam dazu gezwungen sind, eigene Positionen zu entwerfen. Reichenbach hat dies auf die These zugespitzt, dass „nur Dilettanten frei sein können"47. Das bedeutet, dass Menschen allein in den Angelegenheiten Subjekt sein können, in denen die Antworten auf Fragen nicht schon feststehen, sondern allererst noch gesucht und gefunden werden müssen. „Nur auf dem Boden der Imperfektibilität ist es Menschen möglich, sich als Subjekte zu behaupten" 48 .
Indem im Kontext eines erziehenden Unterrichts Fragen des gelingenden Lebens und Zusammenlebens aufgeworfen werden, werden die Heranwachsenden in Situationen hineingezogen, in denen genau das möglich (aber auch erforderlich) ist, was Regenbrecht in Beschreibungen exemplarischen Unterrichts ausgeklammert sieht, nämlich die ,Selbsttätigkeit der Entscheidung'. Wenn Regenbrecht darauf hinweist, dass im Kontext exemplarischen Lehrens und Lernen die ,Selbsttätigkeit der Entscheidung' außen vor bleibe, so macht er darauf aufmerksam, dass hier Fragen ausgeklammert werden, in denen das eigene Urteil der Heranwachsenden gefragt ist. In dieser Lesart ist Regenbrechts Einwand also gar nicht gegen exemplarisches Lehren und Lernen als solches gerichtet. Vielmehr wird deutlich gemacht, dass exemplarischer Unterricht konsequenterweise als erziehender Unterricht begriffen werden muss, soll dem Anspruch der Bildung Rechnung getragen werden.

Wie Heranwachsende sich im Lichte sachlicher Einsichten zu Fragen des Lebens und Zusammenlebens positionieren, muss offengehalten werden, wenn diese als Subjekte adressiert werden sollen. Eine solche Offenheit hat freilich zur Konsequenz, dass die Heranwachsenden uns mit ihren Entwürfen überraschen können, weil sie Vorzugsgesichtspunkte für sich selbst als maßgeblich bestimmen, die wir nicht erwartet und unter Umständen auch nicht erhofft haben. Es mag von daher durchaus so sein, wie Regenbrecht meint, dass es vor allem die Frage nach der Bedeutung sachlicher Einsichten für Fragen des Lebens und Zusammenlebens ist, durch die Heranwachsende als Subjekte adressiert werden können, denn in diesen Fragen treten sich Erzieher und Zu-Erziehende nicht mehr als Lehrer und Schüler gegenüber, sondern als Menschen die gleichermaßen auf der Suche nach 
Orientierung eines gelingenden Lebens und Zusammenlebens sind. ${ }^{49}$

Doch auch an dieser Stelle wäre darauf aufmerksam zu machen, dass das Sich-Verhalten des Einzelnen zum Gelernten nicht unbegrenzt offen ist bzw. offen sein kann, wenn wir davon ausgehen, dass jeder Mensch einen Anspruch hat, eigene Werturteile zu entwerfen und diesen im Handeln zu entsprechen. Es ist von daher nicht weiter verwunderlich, wenn Biesta von einem Unterricht, der über die Qualifizierung und Sozialisierung der Heranwachsenden hinausgeht, nicht nur erwartet, dass Schüler dazu aufgefordert werden, "zu zeigen, wer sie sind und wo sie stehen"50, sondern darüber hinaus die Frage nach überindividuellen Verbindlichkeiten im Miteinanderumgehen als eine zentrale Frage markiert, in deren intergenerationelle Beratung die Heranwachsenden eingeführt werden sollen. Die Frage, „ob das, was wir wünschen für unser Leben und das Zusammenleben mit anderen auch wünschenswert ist" ${ }^{451}$, spielt in Beschreibungen eines erziehenden Unterrichts, die der Herbarttradition zuzurechnen sind, seit jeher eine zentrale Rolle. Entscheidend ist, dass es in diesem Zusammenhang nicht darum geht, Heranwachsende auf bestimmte Antworten hin festzulegen, die Menschen in der Geschichte auf diese Frage gefunden haben, sondern sie in die Suche nach dem Guten und Bösen selbst hineinzuziehen. Dies sieht Biesta offenbar ähnlich: „Die zentrale erzieherische Herausforderung", so Biesta, „besteht nicht einfach nur darin, dem Kind oder dem Schüler mitzuteilen, welche ihrer Wünsche wünschenswert sind, sondern bedeutet vielmehr, dafür zu sorgen, dass diese Frage eine für das Leben des Kindes oder des Schülers bedeutsame Frage wird"52. Der Anspruch lautet also, die Frage nach einem gelingenden Leben und Zusammenleben als Frage zu tradieren, genauer als eine Frage, auf die
Menschen bis heute keine abschließende Antwort gefunden haben - was freilich nicht bedeutet, dass sie überhaupt keine Antworten gefunden hätten. Ein erziehender Unterricht mit Bildungsanspruch führt Heranwachsende in die Frage nach dem Zusammenhang von gelingendem Leben und Zusammenleben sowie in Versuche ihrer Beantwortung ein, und unterstützt sie dabei, eigene Antworten auf die Frage zu finden, zu leben und weiterzuentwickeln.

Es sollte damit deutlich geworden sein, dass sachliche und moralische Restriktionen den unterrichtlichen Anspruch der Subjektwerdung nicht unterminieren. Entscheidend ist, dass Heranwachsenden die Möglichkeit eröffnet wird, sich zu überkommenem Wissen und Können sowie zu tradierten Werten und Normen zu verhalten.

\section{Fazit}

Der in diesem Beitrag entwickelte Gedankengang kann in drei Thesen zusammengefasst werden:

1. Ein Unterricht mit Bildungsanspruch, genauer: ein erziehender Unterricht mit Bildungsanspruch geht über die Aufgaben der Qualifikation und der Sozialisation der nachwachsenden Generation hinaus, indem auch und vor allem die Aufgabe verfolgt wird, Schüler für ein Leben in Selbstbestimmung freizusetzen. Das aber bedeutet, dass diese nicht nur etwas lernen, sondern auch lernen sollen, sich zu Gelerntem zu verhalten.

2. Beschreibungen exemplarischen Lehrens und Lernens können in die Theorie des erziehenden Unterrichts integriert werden, und müssen dies auch, soll eine plausible Antwort auf die Frage gegeben werden, welche Form Inhalte haben müssen, damit diese eine 
die Heranwachsenden von unterrichtlicher Sorge emanzipierende Wirkung entfalten können.

3. Elementares Wissen und Können hat als solches allenfalls eine potentielle Bedeutung für die Lebensführung der Heranwachsenden. Damit dieses tatsächlich eine entsprechende Bedeutung erlangen kann, muss die Hinführung zu elementarem Wissen und Können mit einer Aufforderung zum Entwurf eigener Werturteile verbunden werden. Beschreibungen exemplarischen Lehrens und Lernens unterlaufen so gesehen den eigenen Anspruch, wenn Unterricht nicht zugleich als erziehender Unterricht konzipiert wird - nämlich einen Unterricht zur Darstellung zu bringen, der auf die Ermöglichung von Bildung gerichtet ist.

\section{Anmerkungen}

1 Zur deutschsprachigen Debatte vgl. etwa die Beiträge in Bohl, T./Koch-Priewe, B./Hanke, U./Zierer, K. (Hrsg.): Neuere Ansätze in der Allgemeinen Didaktik. Jahrbuch für Allgemeine Didaktik. Baltmannsweiler: Schneider 2013. Die Frage, was Unterricht ,ist', findet sich behandelt in Vlieghe, J./Zamojski, P.: Towards an Ontology of Teaching. Thingcentred Pedagogy, Affirmation and Love for the World. Cham: Springer 2019. Die Frage, wie Unterricht sein soll, steht im Zentrum der Überlegungen bei Biesta, G.: The Rediscovery of Teaching. New York: Routledge 2017.

2 Vgl. Rucker, Th.: Allgemeine Didaktik als Reflexionsinstanz. Eine wissenschaftstheoretische Grundlegung. In: Zeitschrift für Pädagogik 63 (2017) 5, S. 618-635.

3 Vgl. Derbolav, J.: Das "Exemplarische" im Bildungsraum des Gymnasiums. Düsseldorf: Pädagogischer Verlag Schwann 1957; Scheuerl, H.: Die exemplarische Lehre. Sinn und Grenzen eines didaktischen Prinzips. Tübingen: Niemeyer 1958; Klafki, W.: Das pädagogische Problem des Elementaren und die
Theorie der kategorialen Bildung. 4. durchgesehene und ergänzte Auflage. Weinheim: Beltz 1964; vgl. auch Gerner, B. (Hrsg.): Das exemplarische Prinzip. Darmstadt: Wissenschaftliche Buchgesellschaft 1963.

4 Derbolav, J.: Das Exemplarische als didaktisches Prinzip. In: Th. Ballauff/E. Meyer (Hrsg.): Exemplarisches Lehren - Exemplarisches Lernen. Stuttgart: Ernst Klett 1969, S. 5-8, hier: S. 5 und 8.

5 Vgl. Klafki, W.: Exemplarisches Lehren und Lernen (1983). In: W. Klafki: Neue Studien zur Bildungstheorie und Didaktik. Zeitgemäße Allgemeinbildung und kritisch-konstruktive Didaktik. 6. Auflage. Weinheim und Basel: Beltz 2007, S. 141-161; Klafki, W.: Exemplarisches Lehren und Lernen. In: H. Ludwig/ S.-I. Beutel/K. Kleinespel (Hrsg.): Entwickeln - Forschen - Beraten. Reform für Schule und Lehrerbildung. Weinheim und Basel: Beltz 2006, S. 104-121, hier: S. 105.

6 Vgl. Zierer, K.: Das Prinzip des Exemplarischen: zu Unrecht vergessen (2010). In: K. Zierer: Studien zur Allgemeinen Didaktik. Baltmannsweiler: Schneider 2012, S. 87-100, hier: S. 88f.

7 Vgl. Rucker, Th.: Teaching and the Claim of Bildung. The View from General Didactics. In: Studies in Philosophy and Education 29 (2020) 1, S. 51-69, hier: 54ff.

8 Vgl. Rothland, M.: Allgemeine Didaktik und empirische Unterrichtsforschung als Teilgebiete der Schulpädagogik. In: Die Deutsche Schule 110 (2018) 4, S. 369-382, hier: S. $375 \mathrm{ff}$.

9 Vgl. Rucker, Th.: Bildungstheoretische Didaktik revisited? Über die Möglichkeiten und Grenzen einer bildungstheoretischen Neujustierung der Allgemeinen Didaktik. In: Bildung und Erziehung 72 (2019) 1, S. 104121, hier: S. 108ff.

10 Eine solche Beobachterposition ist z.B. für eine kritisch-skeptische Pädagogik kennzeichnend, in der keine normativen Entwürfe entwickelt, sondern bereits vorliegende Entwürfe auf ihre Voraussetzungen hin thematisiert werden (vgl. Ruhloff, J.: Das ungelöste Normproblem der Pädagogik. Heidelberg: Quelle und Meyer 1979).

11 Vgl. Biesta, G.: What is Education For? On Good Education, Teacher Judgement, and Educational Professionalism. In: European Journal of Eucation 50 (2015) 1, S. 75-87, hier: S. 76. 
12 Gemeint ist damit nicht, dass die Aufgabe von Unterricht endgültig geklärt sein müsste, ehe eine Klärung inhaltlicher oder auch methodischer Problemstellungen beginnen kann. Die Behauptung ist allein die, dass es zumindest einer rudimentären Klärung der Aufgabe von Unterricht bedarf, um inhaltliche bzw. methodische Fragen des Unterrichts sinnvoll formulieren und beantworten zu können.

13 Vgl. Biesta 2015, S. $77 f$.

14 Im dritten Abschnitt dieses Beitrags werde ich auf eine Beschreibung erziehenden Unterrichts zu sprechen kommen, die alle drei der hier genannten Aufgabenstellungen von Unterricht zu berücksichtigen sucht.

15 Im deutschsprachigen Kontext wird mit den Begriffen ,Subjektivierung' oder ,Subjektivation' in der Regel ein Prozess der Unterwerfung durch Macht bei gleichzeitiger Überschreitung zugeordneter Subjektpositionen begriffen. Der Machtcharakter der Subjektgenese wird von Biesta zwar nicht ausgeblendet (vgl. z.B. Biesta, G.: Beyond Learning. Democratic Education for a Human Future. London und New York: Routledge 2006, S. 33ff.), steht aber nicht im Fokus seiner Überlegungen (zu möglichen Verhältnisbestimmungen von ,Bildung' und ,Subjektivation' vgl. Rucker, Th.: Erkenntnisfortschritt durch Problematisierung, oder: Über das Verhältnis von ,Bildung' und ,Subjektivation'. In: Zeitschrift für Pädagogik 60 (2014) 6, S. 908-925, hier: S. 915ff. sowie die Beiträge in Ricken, N./ Casale, R./Thompson C. (Hrsg.): Subjektivierung. Erziehungswissenschaftliche Theorieperspektiven. Weinheim: Beltz 2019).

16 Schäfer, A.: Bildung. In: G. Opp/G. Theunissen (Hrsg.): Handbuch schulische Sonderpädagogik. Bad Heilbrunn: Klinkhardt 2009, S. 44-53, hier: S. 45.

17 Ebd., S. 47

18 Biesta, G.: Bildung and Modernity: The Future of Bildung in a World of Difference. In: Studies in Philosophy of Education 21 (2002) 4/5, S. 343-351, hier: S. 348 und 347.

19 Biesta, G.: Good education in an age of measurement: on the need to reconnect with the question of purpose in education. In: Educational Assessment, Evalusation and Accountability 21 (2009) 1, S. 33-46, hier: S. 41.

20 Biesta, G.: The education-socialisation conundrum or ,Who is afraid of education?'. In: Utbildning \& Demokrati 16 (2007) 3, S. 2536, hier: S. 33.
21 Vgl. Mikhail, Th: Pädagogisch handeln. Theorie für die Praxis. Paderborn: Schöningh 2016, S. 208; Rucker, Th.: Unterricht als Praxis des Gründe-Gebens und NachGründen-Verlangens. Über die methodische Grundstruktur eines Unterrichts mit Bildungsanspruch. In: Pädagogische Rundschau 72 (2018) 4, S. 465-484, hier: S. 474ff.

22 Biesta, G./P. Hannam: The uninterrupted life is not worth living: On religious education and the public sphere. In: Zeitschrift für Pädagogik und Theologie 1 (2019) 2, S. 173-185, hier: S. 177

23 Vgl. Klafki, W.: Didaktische Analyse als Kern der Unterrichtsvorbereitung (1958). In: W. Klafki: Studien zur Bildungstheorie und Didaktik. Durch ein kritisches Vorwort ergänzte Auflage. Weinheim und Basel: Beltz 1975, S. 126-153, hier: S. 134ff.

24 Die Formulierung ,Freisetzung für ein Leben in Selbstbestimmung' soll zum Ausdruck bringen, dass ein Unterricht mit Bildungsanspruch darauf gerichtet ist, Schülern dabei zu helfen, ihr Leben selbstbestimmt führen zu lernen. Dies schließt u.a. mit ein, diese dabei zu unterstützen, sachliche Einsichten und eigene Werturteile zu entwickeln - und damit Voraussetzungen, die - bildungstheoretisch betrachtet - einer Lebensführung überhaupt erst den Charakter einer selbstbestimmten Lebensführung verleihen. Freisetzung bedeutet, dass Unterricht darauf bezogen ist, sich selbst überflüssig zu machen, d.h. Heranwachsende von der unterrichtlichen Sorge durch Lehrer zu emanzipieren. Doch bedeutet die Orientierung an der Emanzipation der Schüler in diesem Sinne nicht notwendigerweise eine Freisetzung für ein Leben in Selbstbestimmung, denn auch ein Unterricht, der nur auf die Einführung der Heranwachsenden in eine gegebene Ordnung gerichtet ist, ist letztendlich darauf bezogen, dass die besagte Einführung überflüssig wird. Kurzum: Schülern die Entwicklung von Selbstbestimmungsfähigkeit zu ermöglichen, schließt zwingend die Emanzipation von unterrichtlicher Sorge mit ein, was umgekehrt nicht der Fall ist. Aus diesem Grund operiere ich mit der Formel ,Freisetzung für ein Leben in Selbstbestimmung', um den Bildungsanspruch von Unterricht zu erfassen.

25 Gruschka, A.: Über die unerledigte „Kategoriale Bildung". In: R. Laging/P. Kuhn (Hrsg.): Bildungstheorie und Sportdidaktik. Ein 
Diskurs zwischen kategorialer und transformatorischer Bildung. Wiesbanden: Springer 2018, S. 49-60, hier: S. 51.

26 Benner, D.: Bildung und Kompetenz. Von der kategorialen Bildung zur Kompetenzorientierung unterrichtlichen Lehrens und Lernens? Überlegungen zur Bedeutung von Wolfgang Klafkis Studien zur Bildungstheorie und Didaktik für eine pädagogisch und kompetenztheoretisch ausgewiesene Didaktik, Unterrichts-undBildungsforschung. In:K.-H. Braun/ F. Stübig/H. Stübig (Hrsg.): Erziehungswissenschaftliche Reflexion und pädagogischpolitisches Engagement. Wolfgang Klafki weiterdenken. Wiesbaden: Springer 2018, S. 73-91, hier: S. 80.

27 Vgl. Klafki, W.: Kategoriale Bildung. Zur bildungstheoretischen Deutung der modernen Didaktik (1959). In: W. Klafki: Studien zur Bildungstheorie und Didaktik. Durch ein kritisches Vorwort ergänzte Auflage. Weinheim und Basel: Beltz 1975, S. 25-45; vgl. Rucker, Th.: Das Problem der Bildungsinhalte. Bildungstheorien unter allgemeindidaktischer Kritik. In: W. Meseth u.a. (Hrsg.): Normativität in der Erziehungswissenschaft. Wiesbaden: Springer 2019, S. 203-221.

28 Vgl. Klafki 1959/1975, S. $35 f$

29 Ebd., S. 44.

30 Klafki, W.: Die Fruchtbarkeit des Elementaren für die Bildungsarbeit der Volksschule. In: Die Bayerische Schule 17 (1964) 25, S. 373379, S. 375.

31 Klafki, W.: Das Problem der Didaktik. Methodologische und bildungstheoretische Voraussetzungen der Didaktik und die Struktur des didaktischen Feldes (1962). In: W. Klafki: Studien zur Bildungstheorie und Didaktik. Durch ein kritisches Vorwort ergänzte Auflage. Weinheim und Basel: Beltz 1975, S. 83-125, hier: S. 120.

32 Klafki 1983/2007, S. 143 f.

33 Klafki 1964, S. 376.

34 Die Differenz zwischen den Begriffen ,Bildungsinhalt' und ,Bildungsgehalt' korrespondiert mit der Differenz zwischen den Begriffen des Exemplarischen und des Elementaren, worauf Klafki explizit hinweist: „Es charakterisiert einen Bildungsinhalt, daß er als einzelner Inhalt immer stellvertretend für viele Kulturinhalte steht [...]. Jene Momente nun, die solche Erschließung des Allgemeinen im Besonderen oder am Besonderen bewirken, meint der Begriff des Bildungsgehaltes. Jeder besondere Bildungsinhalt birgt in sich also einen allgemeinen Bildungsgehalt" (Klafki 1958/1975, S. 134; vgl. Klafki 1962/1975, S. 121f.).

35 Regenbrecht, A. (1964): Zum Verhältnis von Allgemeiner Didaktik und Fachdidaktik an der Pädagogischen Hochschule. In: Pädagogische Hochschule Dortmund (Hrsg.): Die Pädagogische Hochschule. Struktur und Aufgaben. Ratingen: Henn 1964, S. 275-292, hier: S. 288.

36 Biesta 2017, S. 20.

37 Biesta, G.: The Beautiful Risk of Education. London: Paradigm Publishers 2013, S. 4f., 18 und $93 f$.

38 Regenbrecht 1964, S. 288

39 Vgl. Wagenschein, M.: Verstehen lehren. Genetisch - Sokratisch - Exemplarisch (1968). Weinheim und Basel: Beltz. 1999; Berg, H.-C./Gerwig, M./Wildhirt S.: Lehrkunstdidaktik 2013. In: Jahrbuch für Allgemeine Didaktik. Baltmannsweiler: Schneider 2013, S. 11-31.

40 Derbolav, J.: Das Problem einer philosophischen Grundlegung der Pädagogik (1966). In: J. Derbolav: Frage und Anspruch. Pädagogische Studien und Analysen. Wuppertal u.a.: Henn 1970, S. 49-63, hier: S. 61.

41 Klafki, W.: Grundlinien kritisch-konstruktiver Didaktik (1985). In: W. Klafki: Neue Studien zur Bildungstheorie und Didaktik. Zeitgemäße Allgemeinbildung und kritisch-konstruktive Didaktik. 6. Auflage. Weinheim und Basel: Beltz 2007, S. 83-161, hier: S. 123.

42 Für einen Unterricht, der auf das Verstehen einer Sache gerichtet ist, ist es zentral, dass der jeweilige Inhalt in seiner methodischen Konstituiertheit vermittelt und angeeignet wird. Inhalte werden unter Einsatz spezifischer Zugriffe konstituiert und können letztlich nur dann adäquat verstanden werden, wenn dieser Umstand mitreflektiert wird (vgl. Benner, D.: Umriss der allgemeinen Wissenschaftsdidaktik. Grundlagen und Orientierungen für Lehrerbildung, Unterricht und Forschung. Weinheim und Basel 2020, insbesondere S. 195ff.)

43 Klafki, W.: Exempel hochqualifizierter Unterrichtskultur (1999). In: H.C. Berg/U. Aeschlimann (Hrsg.): Die Werkdimension im Bildungsprozess: das Konzept der Lehrkunstdidaktik. Bern: Hep 2009, S. 103-120, hier: S. 115.

44 Vgl. ebd. 
45 Blankertz, H.: Kritische Erziehungswissenschaft. In: K. Schaller (Hrsg.): Erziehungswissenschaft der Gegenwart. Prinzipien und Perspektiven moderner Pädagogik. Bochum: Kamp 1979, S. 28-45, hier: S. 42.

46 Vgl. Rucker, Th.: Erziehender Unterricht, Bildung und das Problem der Rechtfertigung. In: Zeitschrift für Erziehungswissenschaft 22 (2019) 3, S. 647-663.

47 Reichenbach, R.: Demokratisches Selbst und dilettantisches Subjekt. Demokratische
Bildung und Erziehung in der Spätmoderne. Münster: Waxmann 2001, S. 357.

48 Ebd., S. 411.

49 Rucker, Th./Anhalt E.: Perspektivität und Dynamik. Studien zur erziehungswissenschaftlichen Komplexitätsforschung. Weilerswist: Velbrück 2017, S. 13ff.

50 Biesta 2006, S. 29.

51 Biesta 2017, S. 16.

52 Ebd., S. 18. 